\title{
Stereotactic body radiotherapy for localized prostate cancer - 5-year efficacy results
}

\author{
Kristiina Vuolukka ${ }^{1 *}$ (D), Päivi Auvinen ${ }^{1,2}$, Erno Tiainen², Jan-Erik Palmgren', Janne Heikkilä', Jan Seppälä1, \\ Sirpa Aaltomaa ${ }^{3}$ and Vesa Kataja ${ }^{2,4}$
}

\begin{abstract}
Background: The use of stereotactic body radiotherapy (SBRT) as the primary treatment modality in clinically localized prostate cancer (PCa) is emerging. The aim of the study was to analyze the long-term results of PCa patients treated with SBRT.

Methods: This non-selected, real-life patient cohort included 213 patients with localized PCa treated with a robotic SBRT device during 2012-2015.

Results: The median follow-up was 64 months (range, 10-85 months), and all risk-groups were represented as 47 (22.1\%), 56 (26.3\%) and 110 (51.6\%) patients were classified into D'Amico risk stratification of low, intermediate and high-risk groups, respectively. Androgen deprivation therapy (ADT) was administered to $64.3 \%$ of the patients. At cut-off, the biochemical relapse-free survival (bRFS) was 100, 87.5 and $80.0 \%$ for patients at low, intermediate and high-risk ( $p=0.004$ ), and $92.5,84.2$ and $66.7 \%$ for patients with Gleason score $\leq 6,7$ and $\geq 8$, respectively $(p=0.001)$. The actuarial 5-year overall survival (OS) rates were 97.9, 96.4 and 88.6\% in the low, intermediate and high-risk groups, respectively, and at the cut-off, the disease-specific survival (DSS) rate of the whole cohort was high (99.1\%), as only two high-risk patients died due to PCa.

Conclusion: Our present results of SBRT delivered with CyberKnife produced excellent long-term bRFS, OS and DSS outcomes among patients with localized PCa. We conclude that SBRT provides an efficient and convenient treatment option for patients with localized PCa, irrespective of the risk-group.
\end{abstract}

Keywords: Prostate cancer, Radiation therapy, Stereotactic body radiotherapy

\section{Background}

The use of ultra-hypofractionated ( $\geq 5$ Gy / fraction) external beam radiotherapy (EBRT) is emerging as the primary treatment modality in clinically localized prostate cancer $(\mathrm{PCa})$. It can be delivered as stereotactic body radiotherapy (SBRT) by using an image guided robotic or a gantry-based device. The radiobiology of PCa with its low $\alpha / \beta$-ratio and the slow cell proliferation are considered to make PCa sensitive to a high dose per fraction

\footnotetext{
* Correspondence: kristiina.vuolukka@kuh.fi

${ }^{1}$ Cancer Center, Kuopio University Hospital, PO Box 100, Fl-70029 Kuopio, Finland

Full list of author information is available at the end of the article
}

[1-3], and thus hypofractionation advantageous, especially in terms of radiobiological efficacy. Various fractionation schemes have been reported in SBRT literature, but the optimal total dose and dose per fraction are still unknown [4-6].

The majority of the PCa patients included in published SBRT studies have been from low and intermediate-risk groups, with a very good prognosis irrespective of the primary treatment modality $[6,7]$ and rather few of the patients have been at high-risk [8]. In addition, in most of the studies, the follow-up times have been variable; there are only a few with long follow-up times [9-11]. Because of the increasing use of SBRT, there is an

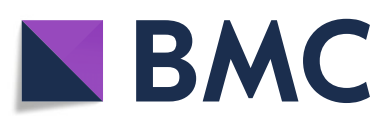

$\mathrm{BMC}$

(c) The Author(s). 2020 Open Access This article is licensed under a Creative Commons Attribution 4.0 International License, which permits use, sharing, adaptation, distribution and reproduction in any medium or format, as long as you give appropriate credit to the original author(s) and the source, provide a link to the Creative Commons licence, and indicate if changes were made. The images or other third party material in this article are included in the article's Creative Commons licence, unless indicated otherwise in a credit line to the material. If material is not included in the article's Creative Commons licence and your intended use is not permitted by statutory regulation or exceeds the permitted use, you will need to obtain permission directly from the copyright holder. To view a copy of this licence, visit http://creativecommons.org/licenses/by/4.0/ The Creative Commons Public Domain Dedication waiver (http://creativecommons.org/publicdomain/zero/1.0/) applies to the data made available in this article, unless otherwise stated in a credit line to the data. 
urgent need to determine long-term results in terms of both efficacy and toxicity.

In the US, the published efficacy and toxicity data have been interpreted to be sufficient for listing SBRT in the AUA-ASCO-ASTRO guidelines as an alternative primary treatment option for low-risk patients and an option in research protocols for intermediate-risk $\mathrm{PCa}$ patients [12]. With respect to high-risk patients, these guidelines are more cautious, because of the very limited data on the efficacy of SBRT in this group. In Europe, the status of SBRT in the treatment of PCa is controversial. In the $\mathrm{PCa}$ treatment guideline issued by the European Association of Urology (EAU), the approach to SBRT is more conservative as its use is restricted only to clinical trials [13] while the European Society for Radiotherapy \& Oncology (ESTRO) endorses the AUAASCO-ASTRO guideline [14].

The first CyberKnife ${ }^{\circ}$ device in Scandinavia was implemented in Kuopio University Hospital (KUH) in April 2012 and since then, SBRT has been one of the treatment options for all PCa patients with localized disease, irrespective of their risk-group, who have been referred to RT for their radical primary treatment. The main exclusion criteria for radical intent SBRT have been the cT4 disease, pelvic nodal disease, a previous pelvic RT and previously operated rectal cancer.

The purpose of this retrospective study was to analyze the long-term efficacy results of the new treatment modality among patients with localized PCa. This study focuses especially on the biochemical relapse-free survival (bRFS) and overall survival (OS) according to iPSA, Gleason score and D'Amico risk-stratification.

\section{Methods}

The patient cohort consists of 218 men with biopsy proven and clinically localized $\mathrm{PCa}$ who were treated with the robotic SBRT device (CyberKnife ${ }^{\bullet}$ Accuray Inc., Sunnyvale, CA) as their primary curative treatment during April 2012 to March 2015. The diagnosis performed by urologists included PSA testing, digital rectal exam and prostate biopsies and the patients were classified according to D'Amico risk-stratification [15]. The patients with D'Amico high-risk disease were imaged with whole-body computer tomography (CT) and bone scan. At the cut-off in June 2019, five patients had been lost to follow-up, and for the purpose of these analyses, they were excluded. This study has been approved by the Ethics Committee of the Northern-Savo Health Care District (895/13.02.00/2019). The primary endpoint of this study was the long-term efficacy of SBRT in terms of bRFS, disease-specific survival (DSS) and OS among patients with localized low, intermediate and high-risk PCa.
The treatment planning and delivery procedures have previously been described in detail [16]. Briefly, for the purposes of image-guidance, four gold fiducials were placed into the prostate under ultrasound guidance. A non-contrast pelvic CT with $1 \mathrm{~mm}$ slice thickness and pelvic magnetic resonance images (MRI) were obtained 7-10 days after the fiducial placement. The MRIs were fused with the CT images to precisely delineate the target volume and the organs at risk (OAR). The clinical target volume (CTV) included the prostate and the proximal seminal vesicles. For high-risk patients with seminal vesicle infiltration (cT3b) in planning MRI scan, the tumorous area of the vesicle was also included into the CTV. To achieve planning target volume (PTV) for patients at low and intermediate-risk, an isotropic expansion of $3-5 \mathrm{~mm}$ was added to CTV. The size of the margin was at the discretion of the radiation oncologist according to the accuracies and possible inaccuracies in the target delineation. Posteriorly, the expansion was always only $3 \mathrm{~mm}$ to protect the anterior rectal wall. To achieve PTV for high-risk patients, an isotropic expansion of $5 \mathrm{~mm}$ was added to CTV, except for $3 \mathrm{~mm}$ in the posterior direction. The OARs included rectum, urethra, bladder, bladder wall, femoral heads, penile bulb and testes and the delineation was performed to the planning images according to KUH protocol.

Initially, all patients were treated to the total dose of $36.25 \mathrm{~Gy}$ in 5 fractions of 7.25 Gy on every other day delivered with CyberKnife ${ }^{\circ}$. In $2013 \mathrm{Katz}$ et al. published a study with a mFU of 60 months showing no additional benefit of the higher total dose of $36.25 \mathrm{~Gy}$ as compared to $35 \mathrm{~Gy}$ among the low-risk and favorable profile (Gleason $3+4=7$ ) intermediate-risk patients [17]. Hence, the treatment protocol in $\mathrm{KUH}$ was changed and the higher total dose was applied only to patients at intermediaterisk with an unfavorable profile (Gleason $4+3=7$ ) and patients at high-risk. The prescribed dose was normalized on average to the $85 \%$ (range, 76-90\%) isodose line. The dose objectives and constraints used for treatment planning are presented in Additional file 1. During a typical 30-45 min treatment session, the fiducials within the prostate were tracked at $15-60 \mathrm{~s}$ intervals by using orthogonal X-ray imaging with the robotic treatment device automatically making the necessary adjustments [16]. Administration of androgen deprivation therapy (ADT) was at the urologist's discretion and based on risk category and prostate size.

During the follow-up time, a typical benign bounce was determined as a prostate-specific antigen (PSA) rise of $0.2 \mathrm{ng} / \mathrm{ml}$ or above with a subsequent, spontaneous decline back to the previous nadir or lower. A biochemical relapse (bR) was defined according to the Phoenix definition, i.e. PSA nadir $+2 \mathrm{ng} / \mathrm{ml}$ [18] and the bRFS was defined as the time from SBRT to the bR. The DSS 
was defined as the time from SBRT to PCa death and the OS as the time from SBRT to death due to any cause. In the statistical analyses, the patients were classified into risk groups according to D'Amico risk stratification [15] and the intermediate-risk patients were subdivided into favorable and unfavorable groups according to the Gleason score $3+4=7$ and $4+3=7$, respectively.

All statistical analyses were performed using the SPSS version 22.0 (SPSS Inc., Chicago, IL). The correlations for categorical factors were calculated by Chi-square test, the univariate analyses were conducted with the Kaplan-Meier method, and the significance of the differences between groups was assessed by the log-rank test. Multivariate analyses including the risk-group, PSA at diagnosis (iPSA), Gleason score, the use of ADT and the total dose were performed by Cox-Regression analysis and a $p$ value $<0.05$ was regarded as statistically significant.

\section{Results}

With a median follow-up (mFU) of 64 months (range, 10-85 months), this study included 213 patients of whom 47 (22.1\%), 56 (26.3\%) and 110 (51.6\%) were classified into low, intermediate and high-risk groups, respectively. The median age of the patients was 70 years (range, 47-86) and the median iPSA was $9.94 \mathrm{ng} / \mathrm{ml}$ (range, $1.3-300.0 \mathrm{ng} / \mathrm{ml}$ ). ADT with varying durations was administered to 137 patients (64.3\%). Detailed patient and treatment demographics are presented in Table 1.

Biochemical relapse was observed in 29 (13.6\%) patients of whom $7(24.1 \%)$ and $22(75.9 \%)$ were classified into intermediate and high-risk groups, respectively, and none in the low-risk group. At mFU of 64 months, the bRFS was $100,87.5$ and $80.0 \%$ for patients at low, intermediate and high-risk $(p=0.004)$, and 92.5, 84.2 and $66.7 \%$ for Gleason score $\leq 6,7$ and $\geq 8$, respectively ( $p=$ 0.001), as depicted in Fig. 1. The ADT administration correlated significantly with the risk of bR, as the bRFS was 96.1 and $81.0 \%$ among the patients treated with ADT compared to those without, respectively $(p=$ 0.003). When subdividing the patients into two groups according to the iPSA i.e. less than $10 \mathrm{ng} / \mathrm{ml}$ vs $10 \mathrm{ng} / \mathrm{ml}$ or higher, the bRFS were 90.7 and $81.7 \%$, respectively, and showing a statistically significant benefit of a low iPSA ( $p=0.046)$. The subdivisions into groups of favorable vs unfavorable profile intermediate-risk disease or to the total irradiation dose of 35 Gy vs 36.25 Gy did not reveal any differences between the groups.

The Cox multivariate regression analysis regarding the risk factors for bR was performed including the total RT dose, use of ADT, Gleason score $(\leq 7, \geq 8)$ and iPSA ( $\leq$ $10 \mathrm{ng} / \mathrm{ml}, \geq 10 \mathrm{ng} / \mathrm{ml}$ ). The Gleason score and the use of
Table 1 Patient and treatment demographics $(n=213)$

\begin{tabular}{|c|c|c|c|}
\hline \multirow[t]{2}{*}{ Patients, $n=213$} & \multicolumn{3}{|c|}{ Risk group $^{a}$} \\
\hline & $\begin{array}{l}\text { Low } \\
n=47\end{array}$ & $\begin{array}{l}\text { Intermediate } \\
n=56\end{array}$ & $\begin{array}{l}\text { High } \\
n=110\end{array}$ \\
\hline \multicolumn{4}{|l|}{ Age $y$} \\
\hline median & 68 & 69 & 71 \\
\hline range & $52-81$ & $47-86$ & $54-86$ \\
\hline \multicolumn{4}{|l|}{ cT-stage n (\%) } \\
\hline T1c-2a & $47(100)$ & $46(82.1)$ & $22(20.0)$ \\
\hline $\mathrm{T} 2 \mathrm{~b}$ & - & $9(16.1)$ & $2(1.8)$ \\
\hline $\mathrm{T} 2 \mathrm{c}-4$ & - & - & $86(78.2)$ \\
\hline NA & - & $1(1.8)$ & - \\
\hline \multicolumn{4}{|l|}{ iPSA ng/ml n (\%) } \\
\hline$<10$ & $47(100)$ & $22(39.3)$ & 39 (35.4) \\
\hline $10-20$ & - & $34(60.7)$ & $41(37.3)$ \\
\hline$>20$ & - & - & $30(27.3)$ \\
\hline median & 7.5 & 11.1 & 11.8 \\
\hline range & $1.9-10.0$ & $1.3-19.0$ & $2.52-300.0$ \\
\hline \multicolumn{4}{|l|}{ Gleason score n (\%) } \\
\hline$\leq 6$ & $47(100)$ & $27(48.2)$ & $33(30.0)$ \\
\hline 7 & - & $29(51.8)$ & $50(45.5)$ \\
\hline$\geq 8$ & - & - & $27(24.5)$ \\
\hline \multicolumn{4}{|l|}{ ADT n (\%) } \\
\hline yes & $13(27.7)$ & $27(48.2)$ & $97(88.2)$ \\
\hline \multicolumn{4}{|l|}{ ADT duration n (\%) } \\
\hline 7 mo or less & $12(25.5)$ & $17(30.4)$ & $28(25.5)$ \\
\hline $12-18 \mathrm{mo}$ & - & $2(3.6)$ & $13(11.8)$ \\
\hline 24 mo or longer & - & $7(12.5)$ & $46(41.8)$ \\
\hline NA & $1(2.1)$ & $1(1.8)$ & $10(9.1)$ \\
\hline \multicolumn{4}{|c|}{ Irradiation dose $\mathrm{n}(\%)$} \\
\hline 35 Gy & $3(6.4)$ & $10(17.9)$ & $22(20.0)$ \\
\hline $36.25 \mathrm{~Gy}$ & $44(93.6)$ & $46(82.1)$ & $88(80.0)$ \\
\hline
\end{tabular}

${ }^{\mathrm{a}} \mathrm{D}^{\prime}$ Amico risk stratification

Abbreviations: iPSA PSA at the time of diagnosis, ADT androgen deprivation therapy, $y$ year, mo month, NA not available

ADT were found to be significant factors predicting biochemical relapse, see Table 2 .

A PSA bounce was observed in $56(26.3 \%)$ patients with a median absolute rise of $0.38 \mathrm{ng} / \mathrm{ml}$ (range, $0.2-$ $2.59 \mathrm{ng} / \mathrm{ml}$ ) with the median time to the first PSA bounce being 17.5 months (range, $6-53$ months). Two or more bounces were observed in $9(4.2 \%)$ patients. The development of a PSA bounce correlated with the risk of bR, as the bRFS was 94.6 and $83.4 \%$ among the patients experiencing a bounce compared to those without, respectively $(p=0.029)$. Correspondingly, more bounces were seen among patients treated with SBRT only compared to patients treated with SBRT in combination with ADT, 39.5 and $19.0 \%$, respectively $(p=0.001)$. The size 

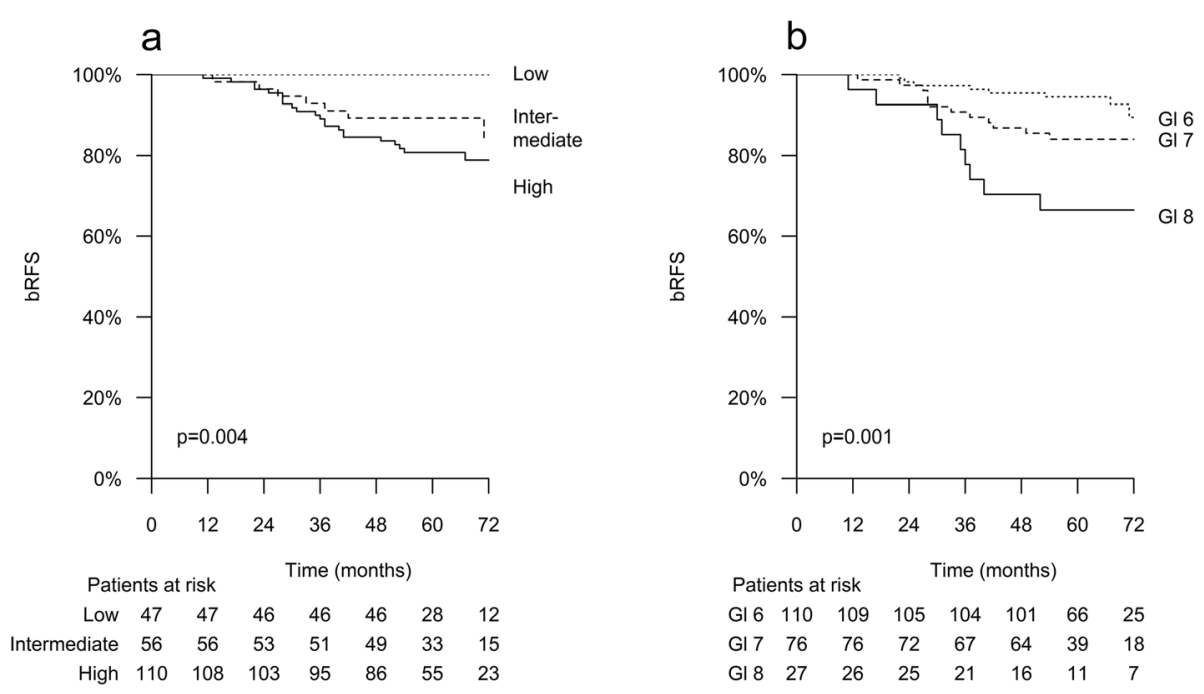

Fig. 1 The biochemical relapse-free survival (bRFS) rates at the median follow-up of 64 months according to the risk-group (a) and to the Gleason score (b)

$(<50 \mathrm{~cm} 3 \mathrm{vs} \geq 50 \mathrm{~cm} 3)$ of the prostate did not correlate with the incidence of a bounce and neither did the delivered total RT dose. Among the non-relapsing patients ( $n=184,86.4 \%$ ), the median iPSA of $9.72 \mathrm{ng} / \mathrm{ml}$ (range, $1.30-300.00 \mathrm{ng} / \mathrm{ml}$ ) declined to a median PSA of 0.24 $\mathrm{ng} / \mathrm{ml}$ (range, $0-3.90 \mathrm{ng} / \mathrm{ml}$ ), $0.22 \mathrm{ng} / \mathrm{ml}$ (range, $0-1.41$ $\mathrm{ng} / \mathrm{ml}$ ) and $0.17 \mathrm{ng} / \mathrm{ml}$ (range, $0-2.10 \mathrm{ng} / \mathrm{ml}$ ) at 12,36 and 60 months, respectively.

At cut-off, the DSS rates were 100, 100 and $98.2 \%$ among the patients at low, intermediate and high-risk, respectively. Altogether 18 (8.5\%) patients died during the follow-up period; two patients died due to PCa, three due to other malignancies and 13 due to other comorbidities or trauma. The OS of the whole cohort was $91.5 \%$. The risk group $(p=0.203)$, the Gleason score $(p=0.460)$, favorable or unfavorable profile among the intermediate-risk patients $(p=0.902)$, the iPSA level $(p=0.193)$, the administration of ADT $(p=0.242)$ or the total irradiation dose $(p=0.777)$ did not exert any statistically significant influence on the OS. Interestingly, the development of a bounce correlated significantly with the OS; at the end of follow-up, all the patients $(100 \%)$

Table 2 Multivariate Cox-regression analysis regarding the pretreatment and treatment characteristics and biochemical relapse

\begin{tabular}{lll}
\hline Variable & HR $(95 \% \mathrm{Cl})$ & $p$ value \\
\hline Gleason score $(\leq 7, \geq 8)$ & $2.35(1.03-5.36)$ & 0.043 \\
iPSA $(<10 \mathrm{ng} / \mathrm{ml}, \geq 10 \mathrm{ng} / \mathrm{ml})$ & $1.36(0.61-3.01)$ & 0.454 \\
Dose $(35$ vs. $36.25 \mathrm{~Gy})$ & $1.10(0.41-2.98)$ & 0.846 \\
ADT (no vs. yes) & $3.72(1.06-13.14)$ & 0.041 \\
\hline
\end{tabular}

Abbreviations: Gy Gray, iPSA initial PSA at diagnosis, $A D T$ androgen deprivation therapy, $H R$ Hazard ratio, $\mathrm{Cl}$ confidence interval with a bounce were alive as compared to $88.5 \%$ of the patients without a bounce $(p=0.010)$. More detailed OS and bRFS results are presented in Table 3.

\section{Discussion}

This study of robotic SBRT treatment among patients with localized PCa shows excellent results. It seems that SBRT can provide represent an efficient and a convenient treatment option irrespective of the $\mathrm{PCa}$ risk-group, as our results compare very well to the results of other primary treatment modalities used in localized PCa [19-25].

Table 3 Detailed efficacy results according to the risk groups

\begin{tabular}{|c|c|c|c|c|}
\hline \multirow{2}{*}{$\begin{array}{l}\text { At mFU of } 64 \\
\text { mo }\end{array}$} & \multicolumn{3}{|c|}{ Risk group ${ }^{\mathbf{a}}$} & \multirow{2}{*}{$\begin{array}{l}p \\
\text { value* }^{*}\end{array}$} \\
\hline & $\begin{array}{l}\text { Low } \\
n=47\end{array}$ & $\begin{array}{l}\text { Intermediate } \\
n=56\end{array}$ & $\begin{array}{l}\text { High } \\
n=110\end{array}$ & \\
\hline \multicolumn{5}{|l|}{ FU mo } \\
\hline median & 61 & 64 & 64 & \\
\hline range & $16-83$ & $12-85$ & $10-84$ & \\
\hline \multicolumn{5}{|l|}{ bRFS } \\
\hline $\mathrm{n}$ & 47 & 49 & 88 & \\
\hline$\%$ & 100 & 87.5 & 80.0 & 0.004 \\
\hline actuarial 3-y, \% & 100 & 92.7 & 88.8 & \\
\hline actuarial 5-y, \% & 100 & 89.1 & 80.0 & \\
\hline \multicolumn{5}{|l|}{ OS } \\
\hline $\mathrm{n}$ & 45 & 53 & 97 & \\
\hline$\%$ & 95.7 & 94.6 & 88.2 & 0.203 \\
\hline actuarial 5-y, \% & 97.9 & 96.4 & 88.6 & \\
\hline
\end{tabular}


In our study, the actuarial 5-year bRFS and OS rates among the high-risk PCa patients were 80.0 and $88.6 \%$, respectively, emphasizing the excellent efficacy of SBRT also among patients with high-risk disease. To the best of our knowledge, this cohort of consecutive high-risk patients is the largest of its kind as the mFU extends over 5 years. There is one published study of a larger pooled cohort $(n=125)$ of high-risk PCa patients treated with $36.25 \mathrm{~Gy}$ in five fractions but with a shorter $\mathrm{mFU}$ time of 3 years. In that study, the estimated 5 -year bRFS of $81 \%$ was similar to our results [26]. The longest follow-up is from a single-institution study including 38 high-risk PCa patients; their 8-year biochemical diseasefree survival rate was $65.0 \%$ [9]. In a review focusing on SBRT in localized high-risk PCa evaluating 13 studies with patient numbers varying from 8 to 125 , the reported efficacy results had varying follow-up times and often the results of the high-risk patients were not reported separately [8]. Thus, the results of the present study add valuable knowledge about the efficacy of SBRT in this particular subgroup.

Since the radiobiology of $\mathrm{PCa}$ is characterized with a very low $\alpha / \beta$-ratio (1.4-1.5 Gy), the total prescribed dose of 35 or 36.25 Gy delivered in less than 2 weeks is comparable to biological 2 Gy equivalent dose of 85-93 Gy. As this theoretical total dose exceeds the total dose of the conventional IMRT treatment at the time (76-78 Gy in $\mathrm{KUH})$, the SBRT protocol was initially adopted also for high-risk PCa patients. The radiobiology of $\mathrm{PCa}$ is so unique that it could, at least partly, explain our good efficacy results also among the high-risk patients. This conclusion is supported by the results of a large doseescalation study of EBRT in which the increase in the total dose correlated statistically significantly with the higher bRFS [20].

In the intermediate-risk group, our actuarial 5-year bRFS of $89.1 \%$ is somewhat lower than expected. In the recently published review and meta-analysis conducted by Jackson et al. [6], the median 5-year bRFS of intermediate-risk patients was $92.1 \%$. In addition, Katz et al. have reported 6-year bRFS of 90.7\% [17]. Patients with intermediate-risk disease pose a challenge for clinicians, since there is clearly heterogeneity among the aggressiveness of the disease within this group. In attempts to achieve more precise prognostic evaluations, it has been proposed to divide the intermediate-risk group into more specific subgroups according to the tumor burden in biopsies [27] or by applying the new Gleason grading issued by the International Society of Urological Pathology consensus conference in 2014 [28]. In several studies, the subdivision of intermediate-risk group has resulted into bRFS of unfavorable profile patients being closer to the results of high-risk patients [11, 17]. However, in our cohort, the subdivision of intermediate-risk patients into favorable and unfavorable-profile groups according to Gleason score did not reveal any clear division within the group. Unfortunately, in this present study, the data of the tumor burden in the diagnostic biopsies was not known. Perhaps in the future, gene assays tracing prognostic molecular biomarkers could potentially help to identify more aggressive tumors from their indolent counterparts in the intermediate-risk group. After all, in our study with the intermediate-risk patients, the 5-year OS was high (96.4\%) and compares well to previous data [11].

The international guidelines are rather unanimous regarding the treatment of SBRT among low-risk patients $[12-14,27]$. Our 5-year bRFS, OS and DSS rates of 100, 97.9 and $100 \%$, respectively, underline the excellent prognosis of patients at low risk. Studies reporting longterm efficacy of SBRT in this subgroup include the previously mentioned large pooled analysis conducted by Kishan et al. with 7-year cumulative bR rate of $4.5 \%$ and OS rate of $91.4 \%$ [11]. The longest reported $\mathrm{mFU}$ after SBRT in low-risk PCa is 9 years in a cohort of 230 patients with 10-year biochemical disease-free survival and DSS rates of 93 and 100\%, respectively [10]. In this subgroup of patients with a very good prognosis, studies with follow-up times surpassing 10 years are needed to identify the optimal total dosing and fractionation as well as the true efficacy of SBRT.

SBRT for localized PCa is feasible also with modern, image-guided linear accelerators [29, 30]. In previously published data, the acute $\mathrm{GU}$ and rectal toxicity have been tolerable with grade 3 adverse events seldom reported and the biochemical control rates have been high [29]. The non-randomized patient cohort published by Nicosia et al. evaluated 149 low and favorable-profile intermediate-risk $\mathrm{PCa}$ patients treated to the total doses of 35 and 37.5 Gy delivered in five consecutive fractions of 7 and $7.5 \mathrm{~Gy}$, respectively [31]. With mFU of 33 months, the 3-year biochemical relapse-free survival and OS rates of the whole SBRT cohort were 100 and 96.4\%, respectively [31]. Another recent study included 178 patients with low and intermediate-risk PCa with the $\mathrm{mFU}$ of 58.9 months [32]. All patients were treated to the total dose of 35 Gy delivered in five fractions on alternate days and ADT was administered to $36(20.1 \%)$ patients. Their 5-year bRFS of $91.6 \%$ and OS of $95.1 \%$ [32] are in line with our 5-year bRFS rates of 89.1 and $100 \%$ and OS rates of 96.4 and $97.9 \%$ among low and intermediate-risk patients, respectively.

A recently published randomized controlled trial (HYPO-RT-PC study) compared SBRT and conventionally fractionated EBRT delivered with linear accelerators in localized PCa and the patients represented either the intermediate or high-risk groups [33]. With the 5 years $\mathrm{mFU}$, the SBRT was reported to be non-inferior to 
conventionally fractionated EBRT, as estimated failurefree survival (FFS) was $84 \%$ in both treatment arms [33]. In the HYPO-RT-PC study, all patients were treated without ADT and the SBRT fractionation (42.7 Gy in seven fractions of $7.1 \mathrm{~Gy}, 3$ fractions per week) was different from that used most in robot assisted SBRT studies, likewise in ours. However, their 5-year FFS of $84 \%$ was in the same order of magnitude as our 5-year bRFS rates of 89 and $80 \%$ among the intermediate and highrisk patients, respectively [33].

The proportion of patients treated with additional ADT was high in our study. Interestingly, this resulted into significantly higher bRFS for the benefit of the combination treatment $(p=0.003)$. In addition, the ADT administration was an independent factor also in the multivariate Cox regression analysis, exerting a strong impact on the risk of bR $(p=0.043)$. Our results differ from previous studies, as in two pooled analyses of SBRT data in localized PCa, the combination of ADT to SBRT has not resulted in a clear benefit $[11,26]$. The difference may be due to the high proportion of high-risk patients in our study. When treating intermediate and high-risk patients with conventional EBRT, ADT plays a significant role. The addition of ADT with varying duration has been shown to be beneficial in biochemical control and OS rates in several studies with EBRT to total doses of 65-70 Gy [34] and high-risk patients do benefit from the addition of ADT, even when treated with present-day high-dose EBRT (76-82 Gy) [35]. The role of ADT in combination to SBRT has not been demonstrated before. In the future, it will be a challenging question if it is ethically appropriate to randomize highrisk patients according to ADT in combination to SBRT. Hence, we propose that it is safest to sustain on the ADT recommendations as in combination with EBRT.

The only factor with a statistically significant correlation to OS was the development of a bounce $(p=$ $0.010)$ and it correlated statistically significantly also to the bRFS $(p=0.029)$. In our study with high proportion of patients on hormonal treatment, also the administration of ADT correlated statistically significantly with the bRFS. As ADT itself keeps the PSA-levels very low among castration-sensitive PCa patients, the medication could, at least partly, explain why fewer bounces were seen among patients treated with SBRT in combination to with ADT. Vice versa, patients with low-risk PCa were treated with SBRT only and hence had a higher odds of experiencing a bounce as well as an excellent prognosis according to all efficacy parameters, including OS.

The intrinsic limitation of our study is its retrospective setting. In addition, there was substantial variation in the duration of the ADT administration and unfortunately, the patient reported quality-of-life data has not been systematically collected. The strengths of the study include the real-life nature of the patient cohort, the homogeneity of the RT planning performed by a single treatment-team and the short recruitment time of 3 years. Nevertheless, these results compare well with the efficacy results achieved by other modern treatment modalities available for localized PCa [20, 22, 23, 31, 32, 36].

\section{Conclusions}

In our study, SBRT delivered with CyberKnife ${ }^{\circ}$ produced excellent 5-year bRFS, DSS and OS rates in all PCa risk groups. These efficacy results were encouraging also among the patients at high-risk and we conclude that SBRT provides an efficient and convenient treatment option for patients with localized PCa in all risk-groups. In the future, the optimal dosing and fractionation of SBRT as well as the role of ADT will need to be determined in randomized studies with sufficient follow-up times.

\section{Supplementary information}

Supplementary information accompanies this paper at https://doi.org/10 1186/s13014-020-01608-1.

Additional file 1. Dose-volume constraints for treatment planning.

\section{Abbreviations}

ADT: Androgen deprivation therapy; bR: Biochemical relapse;

bRFS: Biochemical relapse-free survival; CT: Computer tomography; CTV: Clinical target volume; DSS: Disease-specific survival; EBRT: External beam radiotherapy; ESTRO: European Society for Radiotherapy \& Oncology; FFS: Failure-free survival; iPSA: Initial PSA at diagnosis; mFU: Median followup; MRI: Magnetic resonance image; OAR: Organ at risk; OS: Overall survival; PCa: Prostate cancer; PSA: Prostate specific antigen; PTV: Planned target volume; SBRT: Stereotactic body radiotherapy

\section{Acknowledgements}

The authors acknowledge biostatistician Tuomas Selander for his graphical expertise.

\section{Authors' contributions}

KV has participated in gathering the original patient data, and she was a major contributor in analyzing the data and interpreting of the results. KV was also a major contributor in writing the manuscript. PA participated in the interpretation of the results and contributed to the writing of the manuscript. ET participated in gathering the updated part of the data, in analyzing the results and writing the manuscript. JEP, JH and JS participated in analyzing the data and contributed to the writing of the manuscript. SA and VK have planned and tutored the work and revised the manuscript. All authors have read and approved the final manuscript.

\section{Funding}

The Corresponding Author has received financial support from Instrumentarium Science Foundation, Ida Montin Foundation and Northern Savo Cancer foundation as an unrestricted personal research grant enabling 3 months of full time research work.

\section{Availability of data and materials}

The data that support the findings of this study are available from the patient records of the Kuopio University Hospital, but restrictions apply to the availability of these data, which was used under license for the purposes of the current study only, and so are not publicly available. The data in anonymized form is available from the authors upon reasonable request and with permission of Kuopio University Hospital. 


\section{Ethics approval and consent to participate}

An informed consent has been obtained from each patient before the treatment. This study has been approved by the ethics committee of the Northern Savo Hospital District with the reference number of 895/13.02.00/ 2019.

\section{Consent for publication}

The consent from the patients for the publication was included in the informed consent, the institutional consent for publication has been granted by the Kuopio University Hospital administration. All authors have given the consent for publication.

\section{Competing interests}

The authors declare that they have no competing interests.

\section{Author details}

${ }^{1}$ Cancer Center, Kuopio University Hospital, PO Box 100, Fl-70029 Kuopio, Finland. ${ }^{2}$ University of Eastern Finland, Kuopio, Finland. ${ }^{3}$ Department of Urology, Kuopio University Hospital, PO Box 100, Fl-70029 Kuopio, Finland. ${ }^{4}$ Central Finland Health Care District, Adm Bldg 6/2, Fl-40620 Jyväskylä, Finland.

Received: 4 May 2020 Accepted: 30 June 2020

Published online: 14 July 2020

\section{References}

1. Brenner DJ, Hall EJ. Fractionation and protraction for radiotherapy of prostate carcinoma. Int J Radiat Oncol Biol Phys. 1999;43:1095-101. https:// doi.org/10.1016/s0360-3016(98)00438-6.

2. Proust-Lima C, Taylor JMG, Sécher S, Sandler H, Kestin L, Pickles T, et al. Confirmation of a low $\mathrm{a} / \mathrm{b}$ ratio for prostate cancer treated by external beam radiation therapy alone using a post-treatment repeated-measures model for PSA dynamics. Int J Radiat Oncol Biol Phys. 2011;79:195-201. https://doi.org/10.1016/j.jijobp.2009.10.008.

3. Miralbell R, Roberts SA, Zubizarreta E, Hendry JH. Dose-fractionation sensitivity of prostate cancer deduced from radiotherapy outcomes of 5,969 patients in seven international institutional datasets: $a / b=1.4(0.9-2.2)$. Gy Int J Radiat Oncol Biol Phys. 2012;82:e17-24. https://doi.org/10.1016/j.jijobp. 2010.10.075.

4. Quon HC, Ong A, Cheung P, Chu W, Chung HT, Vesprini D, et al. Onceweekly versus every-other-day stereotactic body radiotherapy in patients with prostate cancer (PATRIOT): a phase 2 randomized trial. Radiother Oncol. 2018;127:206-12. https://doi.org/10.1016/.jradonc.2018.02.029.

5. Zelefsky MJ, Kollmeier M, McBride S, Varghese M, Mychalczak B, Gewanter R, et al. Five-year outcomes of a phase 1 dose-escalation study using stereotactic body radiosurgery for patients with low-risk and intermediaterisk prostate cancer. Int J Radiat Oncol Biol Phys. 2019;1(104):42e49. https:// doi.org/10.1016/j.jijobp.2018.12.045.

6. Jackson WC, Silva J, Hartman HE, Dess RT, Kishan AU, Beeler WH, et al. Stereotactic body radiation therapy for localized prostate cancer: A systematic review and meta-analysis of over 6,000 patients treated on prospective studies. Int J Radiat Oncol Biol Phys. 2019;4(104):778e789. https://doi.org/10.1016/j.jijobp.2019.03.051.

7. Kishan $A U$, King CR. Stereotactic body radiotherapy for low and intermediate-risk prostate cancer. Semin Radiat Oncol. 2017;27:268-78. https://doi.org/10.1016/.semradonc.2017.02.006.

8. Gonzalez-Motta A, Roach M. Stereotactic body radiation therapy (SBRT) for high-risk prostate cancer: where are we now? Pract Radiat Oncol. 2018;8: 185-202. https://doi.org/10.1016/j.prro.2017.11.008.

9. Katz A, Formenti SC, Kang J. Predicting biochemical disease-free survival after prostate stereotactic body radiotherapy: risk-stratification and patterns of failure. Front Oncol. 2016;6(8):168. https://doi.org/10.3389/ fonc.2016.00168.

10. Katz A. Stereotactic body radiotherapy for low-risk prostate cancer: a ten-year analysis. Cureus. 2017;9(9):e1668. https://doi.org/10.7759/ cureus.1668.

11. Kishan AU, Dang A, Katz AJ, Mantz CA, Collins SP, Aghdam N, et al. Longterm outcomes of stereotactic body radiotherapy for low-risk and intermediate-risk prostate cancer. JAMA Netw Open. 2019;2(2):e188006. https://doi.org/10.1001/jamanetworkopen.2018.8006.
12. Morgan SC, Hoffman K, Loblaw DA, Buyyounouski MK, Patton C, Barocas D, et al. Hypofractionated radiation therapy for localized prostate cancer: executive summary of an ASTRO, ASCO and AUA evidence-based quideline. Pract Radiat Oncol. 2018;8(6):354-60. https:// doi.org/10.1016/j.prro.2018.08.002.

13. Mottet N, Cornford P, van den Bergh RCN, Briers E, De Santis M, Fanti S et al. European Association of Urology (EAU) Guidelines on prostate cancer https://uroweb.org/guideline/prostate-cancer/. [Accessed 15 June 2020].

14. The European Society for Radiotherapy \& Oncology (ESTRO). Guideline for prostate cancer. https://elibrary.estro.org/search?type=Activities\&type= Guideline\&q=prostate+cancer. [Accessed 15 June 2020].

15. D'Amico AV, Whittington R, Malkowicz SB, Schultz D, Blank K, Broderick $\mathrm{GA}$, et al. Biochemical outcome after radical prostatectomy, external beam radiation therapy, or interstitial radiation therapy for clinically localized prostate cancer. JAMA. 1998;280:969-74. https://doi.org/10. 1001/jama.280.11.969.

16. Koskela K, Palmgren J-E, Heikkilä J, Virsunen H, Sailas L, Auvinen P, et al. Hypofractionated stereotactic body radiotherapy for localized prostate cancer - first Nordic clinical experience. Acta Oncol. 2017 Jul;56(7):978-83. https://doi.org/10.1080/0284186X.2017.1288923.

17. Katz AJ, Santoro M, Diblasio F, Ashley R. Stereotactic body radiotherapy for localized prostate cancer: disease control and quality of life at 6 years. Radiat Oncol. 2013;8(118). https://doi.org/10.1186/1748-717X-8-118.

18. Roach M, Hanks G, Thames H, Schellhammer P, Shipley WU, Sokol GH, et al. Defining biochemical failure following radiotherapy with or without hormonal therapy in men with clinically localized prostate cancer: recommendations of the RTOG-ASTRO Phoenix consensus conference. Int J Radiat Oncol Biol Phys. 2006;65(4):965-74. https://doi.org/10.1016/j.jirobp. 2006.04.029.

19. Galalae RM, Martinez A, Mate T, Mitchell C, Edmundson G, Nuernberg N, et al. Long-term outcome by risk factors using conformal high-dose-rate brachytherapy (HDR-BT) boost with or without neoadjuvant androgen suppression for localized prostate cancer. Int I Radiat Oncol Biol Phys. 2004; 58(4):1048-55. https://doi.org/10.1016/j.jijobp.2003.08.003.

20. Zelefsky MJ, Yamada Y, Fuks Z, Zhang Z, Hunt M, Cahlon O, et al. Long-term results of conformal radiotherapy for prostate cancer: impact of dose escalation on biochemical tumor control and distant metastases-free survival outcomes. Int J Radiat Oncol Biol Phys. 2008;71(4):1028-33. https:// doi.org/10.1016/j.jprobp.2007.11.066.

21. Hoskin PJ, Rojas AM, Bownes PJ, Lowe GJ, Ostler PJ, Bryant L. Randomised trial of external beam radiotherapy alone or combined with high-dose-rate brachytherapy boost for localised prostate cancer. Radiother Oncol. 2012; 103(2):217-22. https://doi.org/10.1016/.jradonc.2012.01.007.

22. Wang L, Wang B, Ai Q, Zhang Y, Lv X, Li H, et al. Long-term cancer control outcomes of robot-assisted radical prostatectomy for prostate cancer treatment: a meta-analysis. Int Urol Nephrol. 2017;49(6):995-1005. https:// doi.org/10.1007/s11255-017-1552-8.

23. Dearnaley D, Syndikus I, Mossop H, Khoo V, Birtle A, Bloomfield D, et al. Conventional versus hypofractionated high-dose intensity-modulated radiotherapy for prostate cancer: 5 -year outcomes of the randomized, noninferiority, phase 3 CHHiP trial. Lancet Oncol. 2016;17(8):1047-60. https://doi. org/10.1016/S1470-2045(16)30102-4.

24. Incrocci L, Wortel RC, Alemayehu WG, Aluwini S, Schimmel E, Krol S, et al. Hypofractionated versus conventionally fractionated radiotherapy for patients with localised prostate cancer (HYPRO): final efficacy results from a randomized, multicenter, open-label, phase 3 trial. Lancet Oncol. 2016;17(8): 1061-9. https://doi.org/10.1016/S1470-2045(16)30070-5.

25. Arcangeli S, Strigari L, Gomellini S, Saracino B, Petrongari MG, Pinnarò P, et al. Updated results and patterns of failure in a randomized hypofractionation trial for high-risk prostate cancer. Int J Radiat Oncol Biol Phys. 2012;84(5):1172-8. https://doi.org/10.1016/j.ijrobp.2012.02.049.

26. King CR, Freeman D, Kaplan I, Fuller D, Bolzicco G, Collins S, et al. Stereotactic body radiotherapy for localized prostate cancer: pooled analysis from a multi-institutional consortium of prospective phase II trails. Radiother Oncol. 2013;109(2):217-21. https://doi.org/10.1016/j.radonc.2013.08.030.

27. Mohler JL, Srinivas S, Antonarakis ES, Armstrong AJ, Bekelman JE, Cheng H et al. NCCN clinical practice guidelines in oncology, Prostate Cancer, Version 2.2020. https://www.nccn.org/professionals/physician_gls/pdf/prostate.pdf. [Accessed 15 June 2020].

28. Epstein JI, Egevad L, Amin MB, Delahunt B, Srigley JR, Humphrey PA, et al. The 2014 International Society of Urological Pathology (ISUP) consensus 
conference on Gleason grading of prostatic carcinoma: definition of grading patterns and proposal for a new grading system. Am J Surg Pathol. 2016;40(2):244-52. https://doi.org/10.1097/PAS.0000000000000530.

29. Avkshtol V, Dong Y, Hayes SB, Price RA, Sobczak ML, Horwitz EM, et al. A comparison of robotic arm versus gantry linear accelerator stereotactic body radiation therapy for prostate cancer. Res Rep Urol. 2016:8:145-58. https://doi.org/10.2147/RRU.S58262.

30. Seppälä J, Suilamo S, Tenhunen M, Sailas L, Virsunen H, Kaleva E, et al. Dosimetric comparison and evaluation of 4 stereotactic body radiotherapy techniques for the treatment of prostate cancer. Technol Cancer Res Treat. 2017;16(2):238-45. https://doi.org/10.1177/1533034616682156.

31. Nicosia L, Mazzola R, Rigo M, Figlia V, Giaj-Levra N, Napoli G, et al. Moderate versus extreme hypofractionated radiotherapy: a toxicity comparative analysis in low- and favorable intermediate-risk prostate cancer patients. J Cancer Res Clin Oncol. 2019;145(10):2547-54. https://doi.org/10.1007/ s00432-019-02983-3.

32. Franzese C, Badalamenti M, Di Brina L, D'Agostino G, Franceschini D, Comito $T$, et al. Linac-based stereotactic body radiation therapy for low and intermediate-risk prostate cancer. Strahlenther Oncol. 2020. https://doi.org/ 10.1007/s00066-020-01619-7.

33. Widmark A, Gunnlaugsson A, Beckman L, Thellenberg-Karlsson C, Hoyer M, Lagerlund $\mathrm{M}$, et al. Ultra-hypofractionated versus conventionally fractionated radiotherapy for prostate cancer: 5-year outcomes of the HYPO-RT-PC randomised, non-inferiority, phase 3 trial. Lancet. 2019;349(10196):385-95. https://doi.org/10.1016/S0140-6736(19)31131-6.

34. Juloori A, Shah C, Stephans K, Vassil A, Tendulkar R. Evolving paradigm of radiotherapy for high-risk prostate cancer: current consensus and continuing controversies. Prostate Cancer. 2016;2420786. https://doi.org/10. 1155/2016/2420786

35. Zapatero A, Guerrero A, Maldonado X, Alvarez A, San Segundo CG, Cabeza Rodríguez MA, et al. High-dose radiotherapy with short-term or long-term androgen deprivation in localised prostate cancer (DART01/05 GICOR): a randomised, controlled, phase 3 trial. Lancet Oncol. 2015:16(3):320-7. https://doi.org/10.1016/S1470-2045(15)70045-8.

36. Crook J, Marbán M, Batchelar D. HDR prostate brachytherapy. Semin Radiat Oncol. 2020;30(1):49-60. https://doi.org/10.1016/j.semradonc.2019.08.003.

\section{Publisher's Note}

Springer Nature remains neutral with regard to jurisdictional claims in published maps and institutional affiliations.

Ready to submit your research? Choose BMC and benefit from:

- fast, convenient online submission

- thorough peer review by experienced researchers in your field

- rapid publication on acceptance

- support for research data, including large and complex data types

- gold Open Access which fosters wider collaboration and increased citations

- maximum visibility for your research: over $100 \mathrm{M}$ website views per year

At $\mathrm{BMC}$, research is always in progress.

Learn more biomedcentral.com/submissions 